Objectives Describe the socio-professional, clinical and paraclinical characteristics of ACS and their impact on medical fitness to work.

Methods Descriptive retrospective study of cases of patients with history of an ACS, who have consulted the department of occupational medicine in Rabta Hospital, between 2002 and 2020, to evaluate their medical fitness to work.

Results Our series included 43 patients with a male predominance $(81 \%)$ and a mean age of $52.1 \pm 7.2$ years. The main sectors concerned were transport (21\%), construction (19\%) and health (17\%). Patients were mainly employed as unskilled workers (44\%) and professional drivers (28\%). Sixty two per cent of the employees were in a security position, 55\% were exposed to severe weather, and $40 \%$ had a work requiring significant muscular effort. ACS was due to an ST-segment elevation myocardial infarction in 64\% of cases. Coronography showed tight stenosis of the left anterior descending artery (76\%), the right coronary artery $(50 \%)$, and the left circumflex artery (44\%). Coronary angioplasty was indicated in $85 \%$ of cases and coronary artery bypass surgery in $15 \%$ of cases. The mean left ventricular ejection fraction was $54 \%$ and was higher than $60 \%$ in $42 \%$ of cases. Workstation adjustment was proposed in $26 \%$ of patients, while $26 \%$ were transferred to another workstation. Permanent Disability was reported for $20 \%$ of patients, $14 \%$ had a temporary inability to work, $11 \%$ were kept fit to work without restriction and $3 \%$ had received early retirement.

Conclusion The management of ACS should be multidisciplinary, involving the attending and the occupational physician, including an appropriate cardiac rehabilitation programme, in order to facilitate the socio-professional reintegration of the patient.

\section{P-410 PROFILE OF OCCUPATIONAL PNEUMOCONIOSIS IN TUNISIA: ABOUT 14 CASES.}

${ }^{1}$ Amani Dallagi, Nihel Khouja, Jihen Hsinet, Ines Aissa, Saloua Ismail, Siwar Chemingui, Aida Benzarti, Abdelmajid Ben Jemaa. ${ }^{1}$ Faculté de médecine de Tunis, Tunisia

\subsection{6/OEM-2021-EPI.316}

Introduction Pneumoconiosis are interstitial pulmonary pathology induced by prolonged inhalation of inorganic particles, especially encountered in the workplace. Despite their decline in recent years, they have continued to pose an occupational health problem in Tunisia.

Objective To study the socio-professional, clinical and medicolegal characteristics of pneumoconiosis and to assess their impact on ability to work.

Methods Retrospective descriptive study of pneumoconiosis cases referred for professional etiological investigation to the Department of Occupational Medicine of the Rabta Hospital, in Tunis.

Results These were 14 predominantly male patients, with a mean age of $56.57 \pm 12.32$ years and an average professional length of $23 \pm 9.44$ years. They mainly worked in the underground works sector (4 cases), the building materials industry (3 cases) and metallurgy ( 2 cases). They were exposed to free silica mineral dust (11 cases), iron oxide fumes (3 cases) and asbestos dust (2 cases), in the absence of appropriate personal protective equipment for all patients. The time to onset of symptoms from onset of exposure was $27.36 \pm 12.7$ years. They mainly reported exertional dyspnea (14 cases), cough (8 cases) and chest pain (5 cases). Clinically, crackling was objectified in 9 patients and digital clubbing in 3 patients. Paraclinically, radiological signs of pulmonary fibrosis were objectified in 12 cases and emphysema in 4 cases, a restrictive ventilatory disorder was found in 4 cases. Bronchoalveolar lavage was performed in 6 patients and returned pathologically in three cases. Pulmonary disease was at the stage of chronic respiratory failure in one case. Despite the occupational exposure, only 10 cases were declared as compensable occupational diseases: 7 cases of silicosis, 2 cases of asbestosis and 1 case of siderosis. This morbidity justified a workstation layout for 4 patients and permanent incapacity for 2 others.

Conclusion Given the severity of pneumoconiosis and lack of curative treatment, prevention seems essential.

\section{P-413 WORK-RELATED STRESS IN THE OPERATING ROOM: A CROSS SECTIONAL STUDY IN DIFFERENT HEALTH STRUCTURES IN TUNIS}

Nihel Khouja, 'Ines Aissa, Saloua Ismail, Siwar Chemingui, Jihen Hsinet, Amani Dallagi, Aida Benzarti, Abdelmajid Ben Jemaa. ${ }^{1}$ Faculty of Medicine of Tunis, Tunisia

\subsection{6/OEM-2021-EPI.317}

Introduction Working in the operating room exposes to multiple psycho-organizational constraints that can cause negative repercussions on the health of the workers and on their performance at work.

Objectives To study psychosocial and organizational risk factors to which paramedical staffs in operating theatres are exposed, and to assess the level of stress at work and its impact on their health and on their performance at work.

Methods A descriptive cross-sectional study involving paramedical staff in operating rooms working in different health structures in Tunis, and based on a survey including the Karasek and Siegrist models.

Results The study included 91 paramedical staff with a mean age of $30.71 \pm 7.9$ years and a mean professional length of service of $4.80 \pm 5.55$ years. They worked $7 \pm 2.2$ hours/day on a fixed morning schedule $54.9 \%$ of the time and night shifts $23.1 \%$ of the time. They reported 4.5 \pm 1.67 surgical procedures/day. They were dissatisfied with the general conditions of their work in $83.6 \%$ of cases and $60.5 \%$ of them reported being victim of violence. They reported stress-related symptoms such as fatigue (57.8\%), headache $(56.7 \%)$, muscular pain $(53.3 \%)$, anxiety $(45.6 \%)$, mood disorders $(42.2 \%)$, memory $(45.6 \%)$ and concentration disorders (37.8\%). Repercussions on work were repeated delays $(34.4 \%)$, absenteeism $(30 \%)$ and the occurrence of errors $(9.8 \%)$. The assessment of the stress at work showed high psychological demands in $81.3 \%$ of cases and low levels of decision latitude in $49.5 \%$ of cases. $40.7 \%$ was exposed to Efforts-Rewards imbalance. Statistically significant associations were found between job-strain and working in the private sector $(p=0.037)$, between the Effort-Reward imbalance and a number of surgeries $>5 /$ day $(\mathrm{p}=0.024)$ on the one hand and exposure to violence $(p=0.038)$ on the other.

Conclusion A preventive strategy based on an improvement of working conditions must be implemented against the risk factors of stress in the operating room. 